\title{
Role of Synaptic Inhibition in Processing of Dynamic Binaural Level Stimuli
}

\author{
Dan H. Sanes, ${ }^{1,2}$ Brian J. Malone, ${ }^{1}$ and Malcolm N. Semple ${ }^{1,3}$ \\ ${ }^{1}$ Center for Neural Science and Departments of ${ }^{2}$ Biology and ${ }^{3}$ Psychology, New York University, New York, \\ New York 10003
}

\begin{abstract}
We have recently discovered a paradoxical aftereffect associated with inhibition in the gerbil auditory midbrain. Single neurons in the inferior colliculus (IC) were assessed for sensitivity to a virtual motion stimulus produced by modulating the interaural level difference (ILD), a major cue for sound localization. The class of neuron studied was predominantly excited by contralateral stimulation and inhibited by ipsilateral stimulation. Sound pressure level was modulated trapezoidally at the ipsilateral "inhibitory" ear, whereas the contralateral "excitatory" level remained constant. When the inhibitory stimulus was decreased within a range of sound levels that maintained suppression under static conditions, an unexpected discharge was often elicited, apparently because of an aftereffect of synaptic inhibition. In contrast, when the inhibitory stimulus was increased within a range of sound levels that produced only modest suppression under static conditions, neuronal dis-
\end{abstract}

charge was often profoundly suppressed. In many cases the "conditioned enhancement" or "conditioned suppression" persisted for several seconds after the modulation of ILD, and such conditioned responses were influenced by the modulation depth and rate. To test the effect of inhibition in the IC directly, glycine and GABA were pulsed from a glass recording pipette during a constant monaural excitatory stimulus. The acoustically elicited discharge rate was potentiated markedly if preceded immediately by the brief $(0.5-10 \mathrm{sec})$ application of inhibitory transmitter. Collectively, these results revealed unusually long-lasting effects of inhibition that may establish a new range of acoustic cues to which the neuron responds best. This may have broad implications for processing ensuing auditory stimuli.

Key words: acoustic motion; GABA; glycine; interaural level differences; gerbil; inferior colliculus
The significant role played by inhibition in refining the response of auditory neurons to binaural stimuli has been demonstrated clearly by selective acoustic stimulation of inhibitory afferents (Nelson and Erulkar, 1963; Rose et al., 1966; Goldberg and Brown, 1969; Boudreau and Tsuchitani, 1970; Brugge et al., 1970; Casseday et al., 1994; Mogdans and Knudsen, 1994), and by the recent in vivo application of pharmacological manipulations (Faingold et al., 1993; Park and Pollak, 1993a,b, 1994; Le Beau et al., 1996).

In the lateral superior olive (LSO), neurons are dominated by an ipsilateral excitatory projection from the cochlear nucleus and a contralateral glycinergic projection via the medial nucleus of the trapezoid body (Nelson and Erulkar, 1963; Boudreau and Tsuchitani, 1970; Moore and Caspary, 1983; Harnischfeger et al., 1985; Sanes, 1990). This simple architecture gives rise to neurons that are strongly influenced by interaural level differences (ILDs). As one ascends the auditory neuraxis, these LSO-like properties are reiterated, but the complexity of excitatory-inhibitory interactions increases. ILD-sensitive neurons in the dorsal nucleus of the lateral lemniscus (DNLL) receive at least two major inhibitory projections driven by the ipsilateral ear, one direct and the other commissural (Schwartz, 1992; Wu and Kelly, 1996). At the level of the inferior colliculus (IC), inputs converge from multiple ascending glycinergic or GABAergic sources, including the supe-

Received Aug. 11, 1997; revised Oct. 27, 1997; accepted Nov. 3, 1997.

This work was supported by National Institutes of Health Grant DC00540 to D.H.S., National Institutes of Health Grant DC01767 to M.N.S., and the W. M. Keck Foundation (M.N.S.). We thank Sally Thornton, Lisa Wise, Suresh Krishna, and Scott Moulder for comments on this manuscript.

Correspondence should be addressed to Dan H. Sanes, Center for Neural Science, 4 Washington Place, New York University, New York, NY 10003.

Copyright (C) 1998 Society for Neuroscience $0270-6474 / 98 / 180794-10 \$ 05.00 / 0$ rior paraolivary nucleus, the LSO, the ventral nucleus of the lateral lemniscus, and the DNLL (Adams and Mugnaini, 1984; Zook and Casseday, 1987; Saint Marie and Baker, 1990; Shneiderman et al., 1993; Merchan et al., 1994).

Although the consequence of multiple inhibitory projections to the IC is not yet known, it has been proposed that a change in the balance of synaptic excitation and inhibition is instrumental in generating a special sensitivity to dynamic acoustic stimuli (Suga, 1988; Spitzer and Semple, 1993). There is strong evidence that neurons respond differently to binaural cues presented under static and dynamic conditions (Yin and Kuwada, 1983; Reale and Brugge, 1990; Wagner and Takahashi, 1990; Spitzer and Semple, 1991, 1993; Ahissar et al., 1992; Takahashi and Keller, 1992). For example, when a range of interaural time differences (ITDs) is presented separately (i.e., statically), the discharge rate of a delay-sensitive neuron varies systematically with ITD. This ITD tuning profile typically has a clearly defined peak, or best ITD. In contrast, a different tuning profile, with a different peak, emerges if the same range of ITD is presented as a continuous sweep (i.e., dynamically). This physiological evidence (Spitzer and Semple, 1991, 1993) coupled with a recent model of dynamic ITD coding in the IC (Cai et al., 1997) suggests that synaptic inhibition is an important factor in motion processing.

To date, evidence for the involvement of inhibition has been indirect. One approach that could enhance our understanding of this problem is to examine neurons in which inhibition can be manipulated more directly. One class of IC neurons that serves this role is predominantly excited by contralateral stimulation and inhibited by ipsilateral stimulation (EI), and these cells are usually sensitive to ILDs. By focusing on these neurons and generating a 


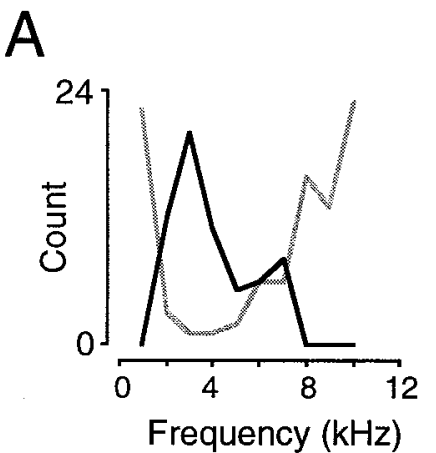

$\mathrm{B}$

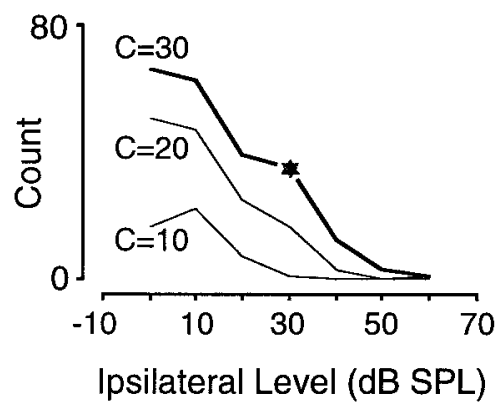

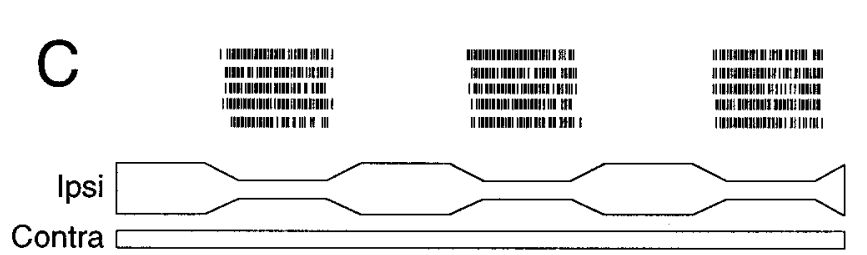

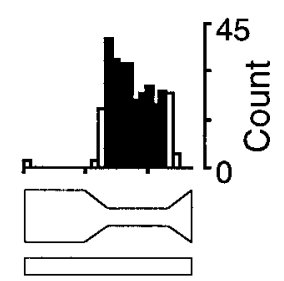

D

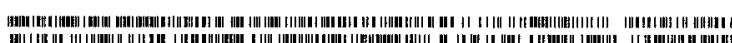

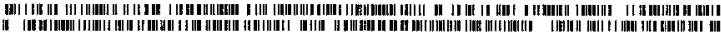

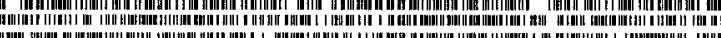

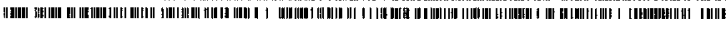

Ipsi Contra

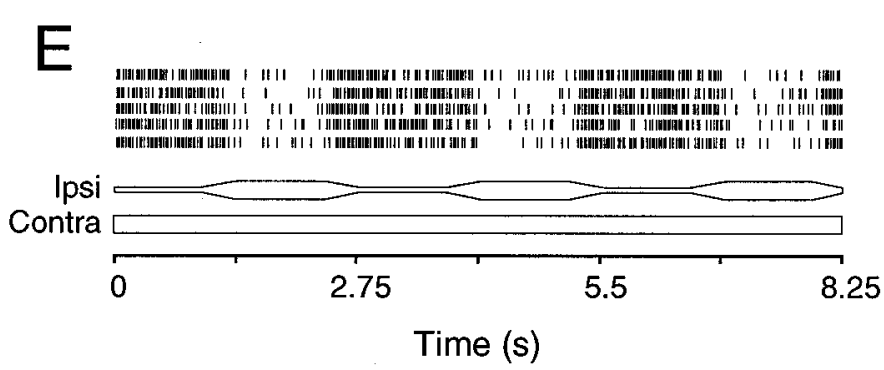

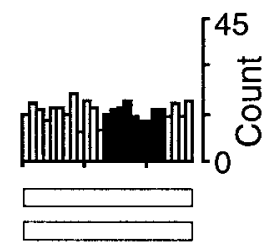

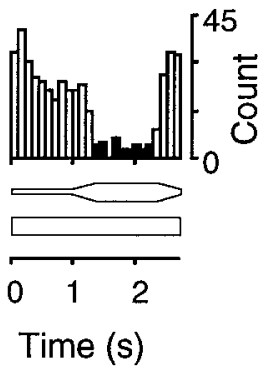

Figure 1. Response of a single IC neuron to static and dynamic sound stimuli. $A$, An excitatory response area was obtained at a single contralateral level (30 dB SPL, black line), and an inhibitory response area was obtained at a single ipsilateral SPL (50 dB SPL, gray line), as described in Materials and Methods. This neuron exhibited a characteristic excitatory and inhibitory frequency of $\sim 3 \mathrm{kHz}$. $B$, Inhibitory rate-level functions were generated for three different contralateral sound levels. The curves can be converted into ILD functions by subtracting ipsilateral from contralateral sound level. The star signifies the target binaural level attained after modulation of ipsilateral SPL. $C-E$, Raster and period histograms of the neuronal response to modulated or static ipsilateral SPL. When ipsilateral level was decreased $(C)$, there was a larger discharge rate at the target SPL, compared with the condition in which ipsilateral SPL remained constant $(D)$. In contrast, when ipsilateral level was increased $(E)$, a smaller discharge rate was elicited at the target SPL, compared with the condition in which ipsilateral SPL remained constant $(D)$. The stimulus levels $(\mathrm{dB})$ were $C$, ipsilateral $(I p s i)=60 \rightarrow 30$, contralateral $($ Contra $)=30 ; D$, Ipsi $=30$, Contra $=30 ;$ and $E$, Ipsi $=0 \rightarrow 30$, Contra $=30$ (unit 95S027.008).

correlate of motion by modulating ILD, we found that ILDsensitive neurons also displayed a special sensitivity to motion stimuli. A possible role for inhibitory mechanisms within the IC was explored, not only with binaural acoustic stimuli but also with a brief application of inhibitory transmitter during monaural excitatory stimulation. Together, these approaches revealed unusually long-lasting effects of inhibition that may establish a new range of acoustic cues to which the neuron responds best. This may have broad implications for processing ensuing auditory stimuli.

\section{MATERIALS AND METHODS}

Surgical preparation. Twenty-nine adult gerbils (Meriones unguiculatus), each with clean ears, were used in these experiments. Animals were anesthetized with one of three protocols: $50 \mathrm{mg} / \mathrm{kg}$ ketamine and 350 $\mathrm{mg} / \mathrm{kg}$ choral hydrate $(n=9) ; 50 \mathrm{mg} / \mathrm{kg}$ ketamine and $50 \mathrm{mg} / \mathrm{kg}$ pentobarbital $(n=18)$; or $0.3 \mathrm{mg} / \mathrm{kg}$ Fentanyl, $10 \mathrm{mg} / \mathrm{kg}$ Fluanisone, and 5 $\mathrm{mg} / \mathrm{kg}$ Midazolam $(n=2)$. Supplemental anesthesia eliminated responsiveness to nociceptive stimuli (e.g., toe pinch) throughout the experiment. Because the effects of virtual motion stimuli were demonstrable under all anesthetic protocols, the data were combined. All procedures were reviewed and approved by the New York University Institutional Animal Care and Use Committee. Animals underwent tracheotomy, and standard surgical procedures were used to expose the tympanic annuli and the dorsal surface of the brain just rostral to the lambdoid suture.

Electrophysiology and auditory stimulation. Metal ear probes were sealed to each annulus, and the closed acoustic system was calibrated from $100 \mathrm{~Hz}$ to $40 \mathrm{kHz}$ (Bruel and Kjaer 0.5 inch microphone), as described previously (Spitzer and Semple, 1993). Acoustic stimuli were generated via digital signal-processing hardware and associated Macintosh-based software (MALab, Kaiser Instruments). Analog biological data were digitized for discrimination in time and amplitude, and all discharge times were stored (with a resolution of $1 \mu \mathrm{sec}$ ) for subsequent analysis.

Platinum-plated tungsten microelectrodes (Microprobe or Ainsworth, $\sim 2 \mathrm{M} \Omega$ ) were advanced (Burleigh Microdrive) ventrocaudally through the overlying cerebral cortex toward the IC, and a single neuron was isolated. We searched in particular for neurons that were excited contralaterally and inhibited ipsilaterally, and this report is limited to describing properties of these EI neurons. For each EI neuron, the contralateral excitatory and ipsilateral inhibitory frequency ranges were determined. Static tone pulses $(200 \mathrm{msec}$ tones, $700 \mathrm{msec}$ trial periods, and 10 repetitions) were then presented at the excitatory characteristic frequency $(\mathrm{CF})$ of the neuron, and spike count was assessed as a function of monaural contralateral level and as a function of ILD.

Dynamic level stimuli were delivered to the ipsilateral "inhibitory" ear while a constant sound pressure level (SPL) was maintained at the contralateral excitatory ear, resulting in a modulation of ILD. Long duration stimuli were used to assess dynamic effects. In one protocol, ILD was modulated trapezoidally with 1 sec steady state components (for stimulus configuration, see Fig. $1 C, D$ ). This regimen was delivered either repetitively for several seconds or as individual trials with a rest interval of a few seconds between each trial. The ipsilateral sound level was modulated at depths of $\pm 5-40 \mathrm{~dB}$ and at rates of 5-120 dB/sec. The time that discharge rates remained elevated or suppressed after a modulation was measured from $12 \mathrm{sec}$ trials, and the histograms were subjected to spline fits $(\lambda=0.01)$ for comparison. For example, the duration of an enhanced response was delimited by the time points at which the spline fit intersected with the spline fit from control trials.

Conjoint recording and pressure ejection. A second procedure was used to examine the role of inhibition. Monaural contralateral "excitatory" stimuli were delivered during local application of inhibitory amino acids through the glass recording pipette. These electrodes $(20-50 \mathrm{M} \Omega)$ were filled with $0.4 \mathrm{M} \mathrm{NaCl}$ and $10 \mathrm{~mm}$ GABA plus $10 \mathrm{~mm}$ glycine. Both amino acids were 

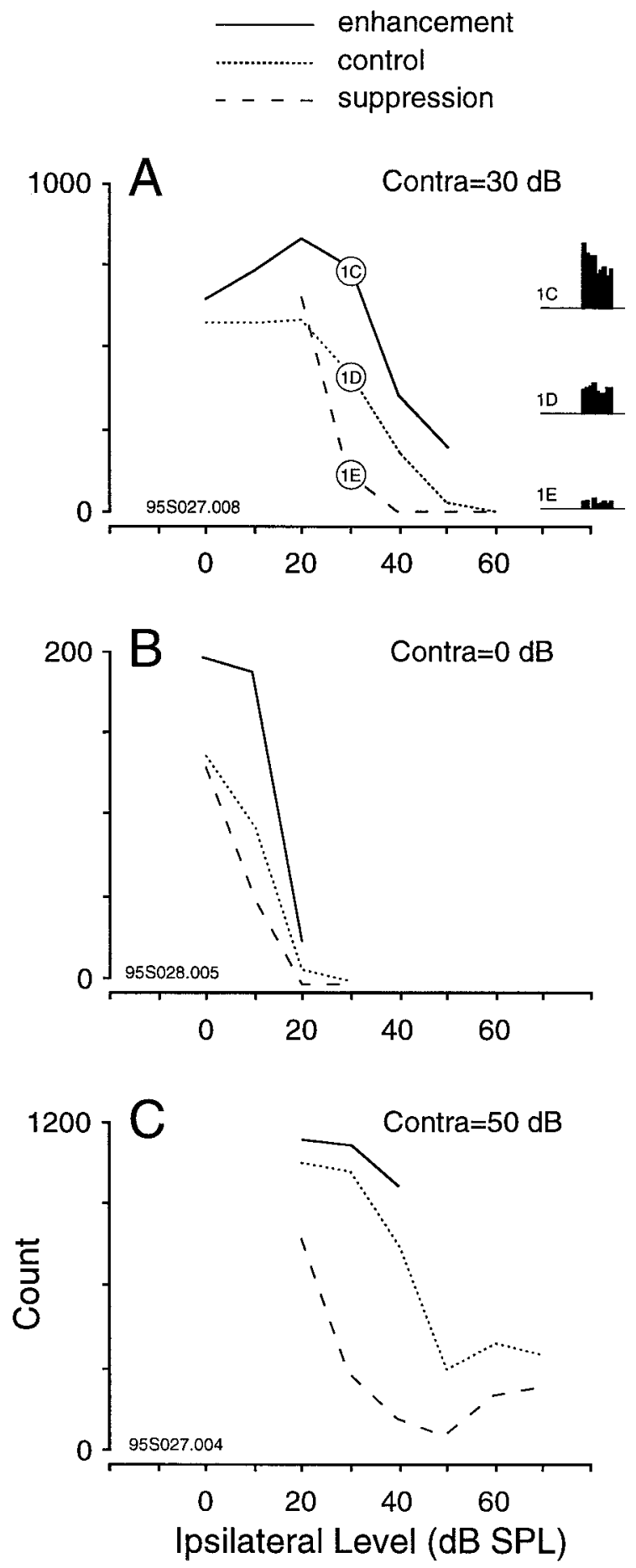

Figure 2. Discharge count obtained for three different IC neurons after modulation to several binaural target SPLs. The $1 \mathrm{sec}$ time window for analysis is shown to the right in $A$. The results demonstrate that an unexpected response was only obtained after modulation to specific binaural levels. In each case the solid line plots the response after a decrease of ipsilateral SPL (conditioned enhancement), the dotted line shows the response when ipsilateral SPL was not modulated (control trials), and the dashed line plots the response after an increase of ipsilateral SPL (conditioned suppression). $A$, This neuron displayed greatest enhancement and suppression when ipsilateral SPL was modulated to 30 $\mathrm{dB}$. In contrast, increasing ipsilateral SPL to $20 \mathrm{~dB}$ produced no suppression, and decreasing ipsilateral SPL to $0 \mathrm{~dB}$ produced no enhancement. Three of the raster and period histograms for this neuron are shown in Figure $1 C-E$, as denoted in the circled points on the plot. Depth, $\pm 30 \mathrm{~dB}$. also delivered in isolation at $10 \mathrm{~mm}$. The electrode holder pressure port was coupled to a valve (Picospritzer), permitting drug delivery (3-60 psi, 0.5-10 sec) that could be triggered at any time during the auditory trial.

\section{RESULTS}

\section{Conditioned enhancement and suppression}

The principal finding was that modulation of inhibitory sound level resulted in unexpected changes in discharge rates. In the initial set of experiments, a neuron was first characterized in a conventional manner using $200 \mathrm{msec}$ static tone pulses. The contralateral excitatory and ipsilateral inhibitory tuning properties are shown for one such neuron in Figure 1A. The range of inhibition was assessed by presenting a matrix of static ILD combinations at $\mathrm{CF}$ and can be illustrated by plotting the elicited spike count as a function of ipsilateral level for one or more fixed contralateral levels (Fig. 1B).

To assess the effect of virtual acoustic motion, time-varying ILD stimuli were generated by maintaining a constant contralateral (excitatory) level and trapezoidally modulating the ipsilateral (inhibitory) level. The ipsilateral stimulus was set initially to a level that suppressed the contralaterally evoked discharge rate partially or completely. Representative raster plots obtained during modulation and control trials (five trials per stimulus condition) are presented in Figure $1 C-E$, with the stimuli schematized beneath each response. The ipsilateral stimulus was increased trapezoidally or decreased to a target level of $30 \mathrm{~dB}$ SPL, a value that was found to suppress discharge by $\sim 50 \%$ when short binaural tone pulses were used (for contralateral, $30 \mathrm{~dB}$ SPL; Fig. 1B, star). The modulation was repeated several times within a trial, and discharges were sorted into the period-based response histogram shown to the right of each set of rasters. The target SPLs differed from neuron to neuron. The most effective target levels often corresponded to the steep portion of the ILD function.

When the ipsilateral stimulus was modulated repetitively from a relatively high sound level (60 dB SPL), at which the binaural balance was strongly inhibitory, to a lower target SPL ( $30 \mathrm{~dB})$, the neuron exhibited an enhanced discharge rate at the target, hereafter referred to as "conditioned enhancement" (Fig. 1C). In particular, this response was found to be enhanced when compared directly with control trials in which stimuli were delivered for the same duration but without trapezoidal modulation (Fig. $1 D$ ). The control trials were folded on a $2.75 \mathrm{sec}$ period to match the period of modulation in the dynamic conditions. The discharge rate remained elevated for some time after the trapezoidal decrease in ipsilateral sound level. This and other characteristics are described in greater detail below. When ipsilateral level was modulated repetitively from a relatively low sound level $(0 \mathrm{~dB}$ SPL) to the same target SPL $(30 \mathrm{~dB})$, the neuron exhibited a lower discharge rate than expected, hereafter referred to as "conditioned suppression" (Fig. 1E). Once again, the conditioned suppression lasted well beyond the modulation component.

A consistent feature of such conditioned responses to dynamic stimuli is that they were elicited by a limited range of binaural combinations. This point is illustrated for three different neurons in Figure 2. In the first example, the same neuron considered in

$\leftarrow$

$B$, This neuron displayed greatest enhancement when ipsilateral SPL was modulated to $10 \mathrm{~dB}$ but did not display significant suppression for any of the stimuli tested. Depth, $\pm 20 \mathrm{~dB}$. $C$, This neuron displayed greatest suppression when ipsilateral SPL was modulated to 30 or $40 \mathrm{~dB}$ but did not display enhancement for any of the stimuli tested. Depth, $\pm 30 \mathrm{~dB}$. 


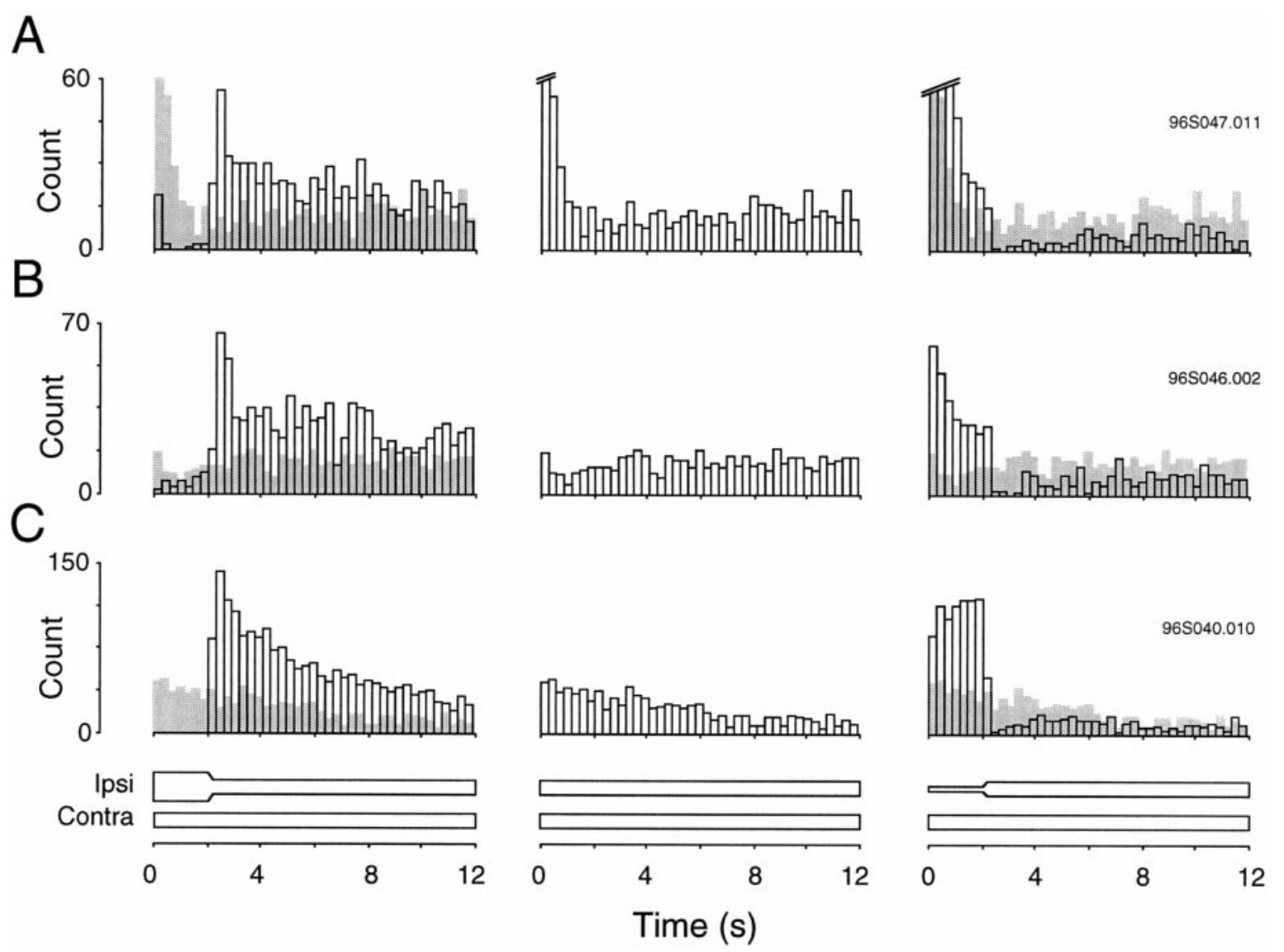

Figure 3. Time course of conditioned enhancement or suppression after acoustic motion stimuli is shown for three IC neurons. Effects for each neuron are shown under three conditions: when ipsilateral SPL is decreasing (left), constant (center), and increasing (right). Each neuron exhibited a long-lasting enhanced (left) or suppressed (right) response after the virtual motion component, compared with the response to an unmodulated control stimulus (middle). The control response is superimposed as light gray bars for comparison. The frequency and sound levels used were $A$, ipsilateral $($ Ipsi) $=$ $40 \rightarrow 20 /$ contralateral $($ Contra $)=30$, Ipsi $=20 /$ Contra $=30$, Ipsi $=0 \rightarrow 20 /$ Contra $=30, \mathrm{CF}=4.5 \mathrm{kHz} ; B$, Ipsi $=70 \rightarrow 40 /$ Contra $=10$, Ipsi $=40 /$ Contra $=$ 10, Ipsi $=10 \rightarrow 40 /$ Contra $=10, \mathrm{CF}=8 \mathrm{kHz}$; and $C$, Ipsi $=70 \rightarrow 50 /$ Contra $=10$, Ipsi $=50 /$ Contra $=10$, Ipsi $=30 \rightarrow 50 /$ Contra $=10$, CF $=21 \mathrm{kHz}$.

Figure 1 was exposed to a sequence of stimuli in which the contralateral level remained constant while the ipsilateral target SPL was systematically varied (Fig. $2 A$ ). For example, the ipsilateral level was modulated trapezoidally from 90 to $60 \mathrm{~dB}$, then 80 to $50 \mathrm{~dB}$, and so forth, until a set of responses was obtained. In Figure $2 A$, the response magnitude is expressed as a function of the ipsilateral target sound level for $1 \mathrm{sec}$ after the modulation component $( \pm 30 \mathrm{~dB})$. For control trials, the discharge rate was acquired from the identical peristimulus time window (from 1.375 to $2.375 \mathrm{sec}$ ). The plot obtained after trapezoidal decrease of inhibitory level shows that discharge rate was greater than expected for a delimited range of ipsilateral target levels. In this case, an ipsilateral modulation from 60 to $30 \mathrm{~dB}$ SPL produced the best conditioned enhancement. The plot obtained after trapezoidal increase of ipsilateral level shows a lower than expected discharge rate when ipsilateral level was modulated from 0 to 30 $\mathrm{dB}$ SPL. Note that the plot from control trials was similar to the ILD curve obtained with $200 \mathrm{msec}$ tone pulses (Fig. 1B).

A summary plot for a neuron that exhibited conditioned enhancement but not conditioned suppression is shown in Figure $2 B$. In contrast, a neuron that displayed conditioned suppression but not conditioned enhancement is shown in Figure 2C. Approximately $65 \%$ of the single EI neurons that were tested $(n=63$ of 97) displayed conditioned enhancement, and $\sim 80 \%$ displayed conditioned suppression ( $n=41$ of 51 ; fewer neurons were tested with increasing ipsilateral level). A failure to display conditioned enhancement in response to virtual motion stimuli was commonly associated with a rapid adaptation of the contralaterally evoked response.

\section{Kinetics of response}

Dynamic conditioning of responses to ILD stimuli was not restricted to the time during which sound level changed. Rather, the enhanced or suppressed discharge rates often outlasted the dynamic component by several seconds. To evaluate the recovery kinetics after modulation of the ipsilateral level, the discharge pattern was monitored for $10 \mathrm{sec}$. Figure 3 illustrates the response of three EI neurons when the ipsilateral level decreased (left), remained constant (middle), and increased (right). For comparative purposes, the response histogram to constant ipsilateral level (control trial) is superimposed in gray on each modulation histogram. In each case, decreasing ipsilateral level produced a response that remained higher than the control histogram for several seconds. Likewise, increasing ipsilateral level produced a response that remained lower than the control histogram for several seconds. The mean duration of conditioned enhancement was $5.5 \pm 0.8$ sec $(n=19)$, and the mean duration of conditioned suppression was $5.3 \pm 0.9 \sec (n=14)$. 


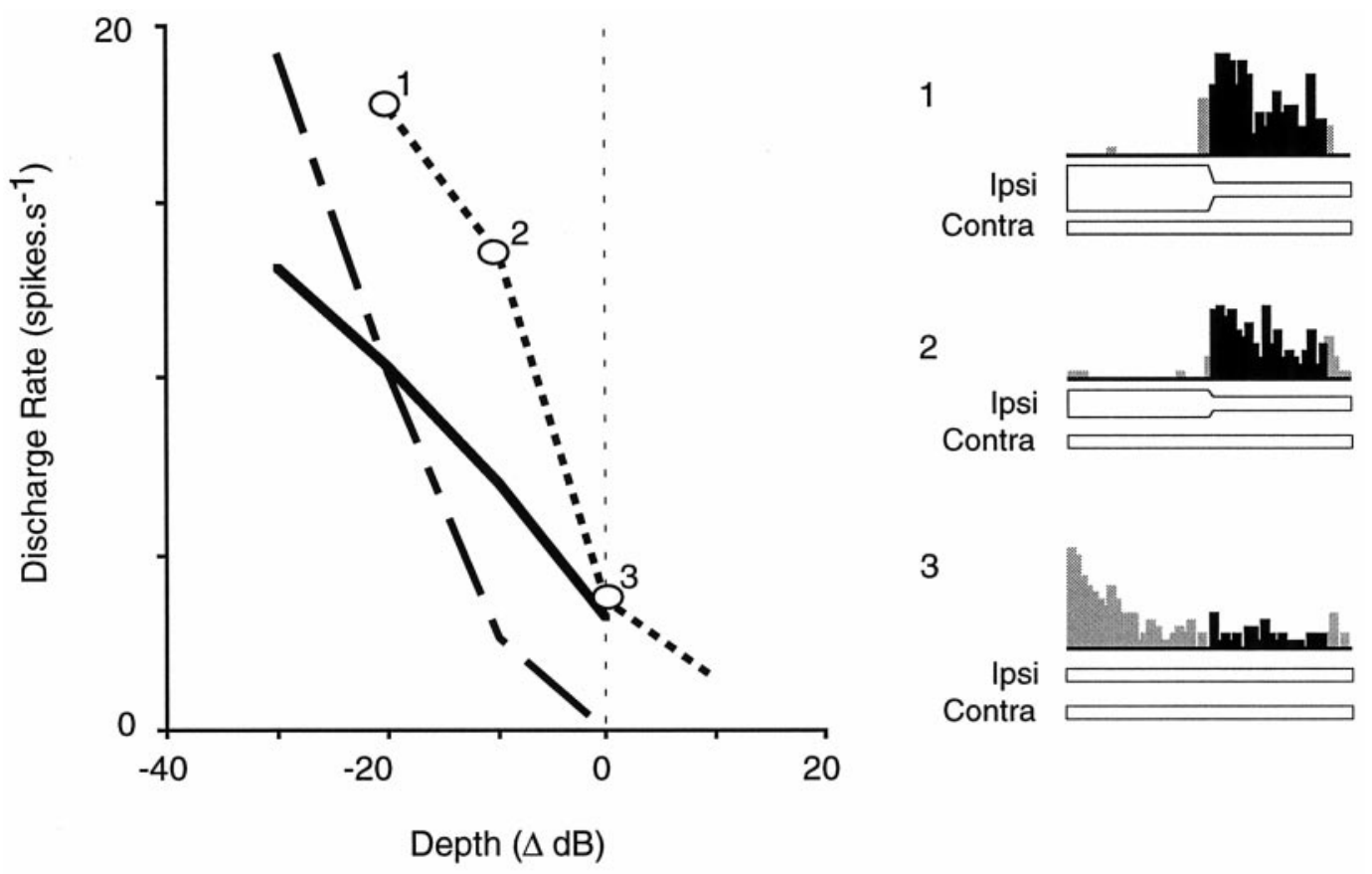

Figure 4. The effect of modulation depth on conditioned enhancement is shown for three IC neurons. In each case, discharge magnitude increased when ipsilateral level was modulated by $-10 \mathrm{~dB}$, and the response was maximal at a depth of -20 to $-30 \mathrm{~dB}$. When depth equals 0 , there is no ipsilateral modulation.

\section{Depth of modulation}

Most neurons were tested with ipsilateral stimuli the level of which changed by \pm 20 or $30 \mathrm{~dB}$. To characterize the influence of modulation depth in more detail, several neurons were tested with depths ranging from +40 to $-40 \mathrm{~dB}$, while keeping the ipsilateral target level and modulation rate constant. Figure 4 shows the discharge rate of three EI neurons as a function of the depth of modulation. Negative values designate a decrease in ipsilateral level, and positive values designate an increase in ipsilateral level. The maximum conditioned enhancement was usually observed for depths of -10 and $-20 \mathrm{~dB}$. In a few neurons, a depth of $-5 \mathrm{~dB}$ was sufficient to produce conditioned enhancement (data not shown), suggesting that the coding properties described herein may be relevant to the dynamic ILD changes that would occur across the small head of a rodent. For the cases shown in Figure 4, the parameters were optimized to illustrate conditioned enhancement (i.e., the ipsilateral target level was chosen to produce relatively strong inhibition and low discharge rate, at a depth of 0 ). Thus, conditioned suppression is not illustrated. When ipsilateral level was adjusted to produce relatively weak inhibition at a depth of $0 \mathrm{~dB}$, then the maximum conditioned suppression was usually observed for depths of +10 and $+20 \mathrm{~dB}$ (data not shown).

\section{Rate of modulation}

Most neurons were tested with a modulation rate of $80 \mathrm{~dB} / \mathrm{sec}$. At this rate, our most commonly used modulation depths of 20 and 30 $\mathrm{dB}$ required modulation times of 125 and $375 \mathrm{msec}$, respectively. In subsequent experiments modulation rate was varied to determine, in particular, whether conditioned enhancement could be observed for very slow virtual motion stimuli. As shown in Figure 5, some neurons exhibited an enhanced response to downward modulation of the inhibitory stimulus for rates as low as $5 \mathrm{~dB} / \mathrm{sec}$ (e.g., a $6 \mathrm{sec}$ modulation duration at a depth of $-30 \mathrm{~dB})$. Conditioned enhance- ment reached a greater maximum response at more rapid rates of modulation, becoming best at $\sim 30-60 \mathrm{~dB} /$ second.

\section{Experimental isolation of ipsilateral inhibition}

Our typical stimulus paradigm activated both contralateral and ipsilateral afferents, and changes in either pathway could have contributed to the effect. To examine independently the role of inhibition, exclusively ipsilateral stimuli were presented to some spontaneously active EI neurons. As shown in Figure 6, ipsilateral stimuli decreased spontaneous discharge, as expected, during the first second of stimulation. When the inhibitory sound level was modulated down, there was a significant enhancement in the neurons discharge rate, relative to the normal spontaneous discharge level (gray histogram). Thus, conditioned enhancement did not necessarily require the use-dependent modulation of contralateral excitatory synapses.

\section{Experimental isolation of contralateral excitation}

The above observations indicated that the conditioned enhancement consequent to virtual motion stimulation could be mediated by ipsilaterally evoked inhibition. Confounding this interpretation, stimulation of the ipsilateral ear recruits inhibitory and excitatory synapses at several loci in the auditory brain stem. Any or all of these synapses might be contributing to conditioned enhancement. As a first step toward clarifying this circuit, we focused on inhibition within the IC. Using a glass recording pipette (see Materials and Methods), we first identified a neuron that displayed conditioned enhancement in response to a virtual motion stimulus (Fig. 7A). Single-unit recordings were maintained while the contralateral acoustic stimulus was paired with short pressure pulses of GABA-glycine delivered from the same pipette in lieu of the ipsilateral acoustic stimulus. The pipette characteristics were designed to ensure that single-neuron recordings were comparable to those obtained with metal electrodes, 


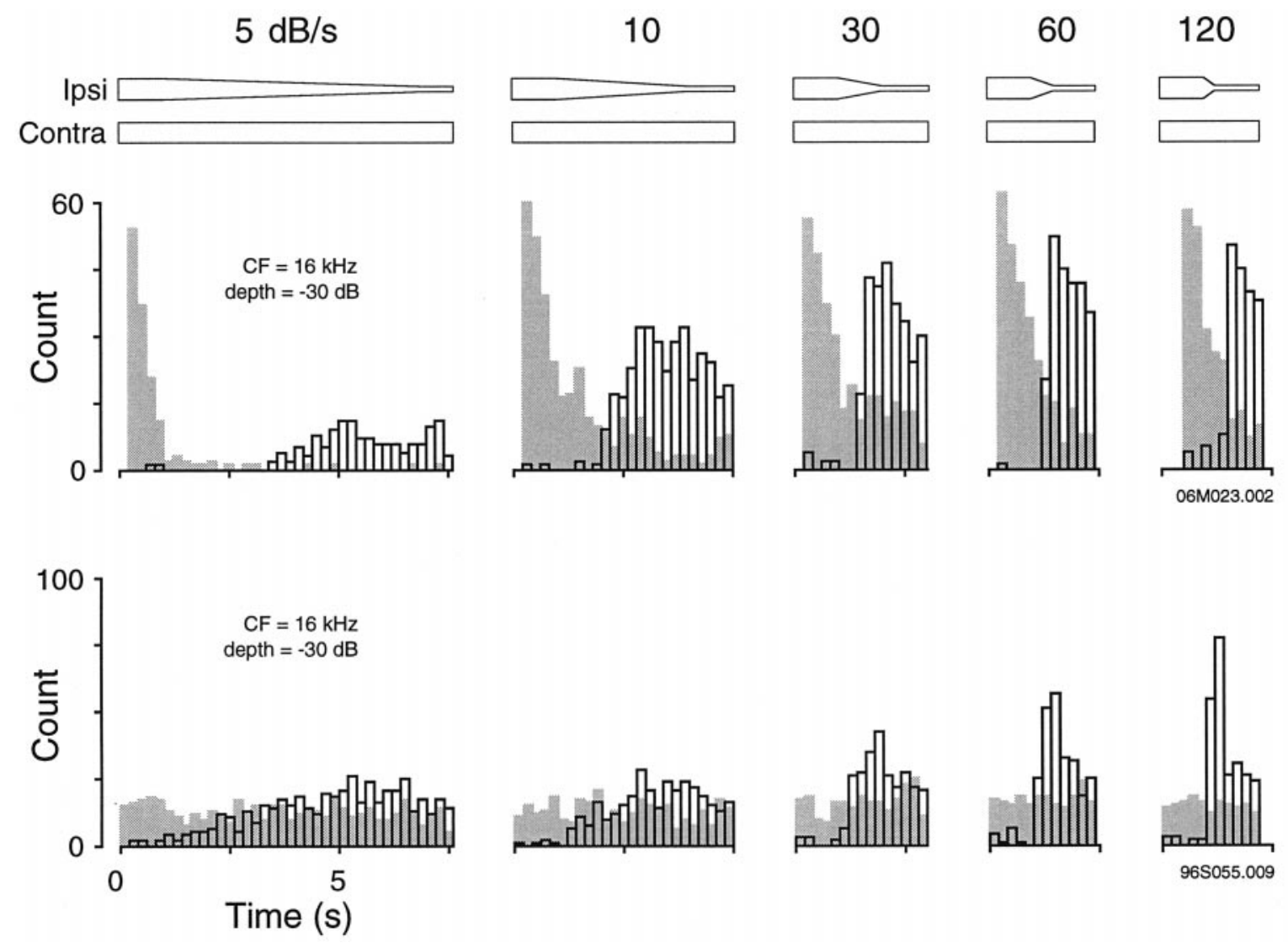

Figure 5. The influence of modulation rate on conditioned enhancement. The response of two IC neurons is shown for modulation rates from 5 to 120 $\mathrm{dB} / \mathrm{sec}$. The control response obtained with constant binaural SPL is shown for each histogram (gray bars). The modulation period during which ipsilateral amplitude is decreasing is shown by the schematic above the histograms. The first neuron (top) exhibited significant enhancement even at the slowest rate of modulation, $5 \mathrm{~dB} / \mathrm{sec}$. The first bar of the control histogram was removed for clarity. The second neuron (bottom) displayed significant enhancement at a rate of $10 \mathrm{~dB} / \mathrm{sec}$.

and that the fluid volume ejected did not perturb the quality of recording (Fig. $7 B$ ). When a 5 sec pulse of GABA-glycine was applied to the neuron during contralateral stimulation, discharge was blocked but resumed almost immediately at the pulse offset (Fig. $7 B$ ), presumably because of active reuptake. As illustrated in Figure $7 C$, the neuron displayed a significant increase in the contralaterally evoked discharge immediately after the GABAglycine pulse was terminated. By comparison, a much lower discharge rate was elicited by the contralateral stimulus immediately before drug was ejected. Thus, local inhibition of the IC neuron by GABA-glycine was an effective substitute for ipsilateral ear stimulation.

Many IC neurons were not clearly inhibited by ipsilateral sound stimulation and were thus excluded from the binaural portion of this study. Nevertheless, several of these neurons did exhibit acoustically evoked inhibition. For example, discharge rate was a nonmonotonic function of contralateral stimulus level in many neurons. Therefore, the effects of GABA-glycine application were assessed in conjunction with several types of contralateral stimuli. As shown in Figure $8 A$, the contralaterally evoked discharge of one neuron first increased and then decreased during a $20 \mathrm{sec}$ tonal stimulus (gray bars). Nevertheless, it was possible to enhance the excitatory response when a pulse of GABA-glycine was delivered $10 \mathrm{sec}$ after the onset of the $20 \mathrm{sec}$ tone (open bars). When a 1 sec pulse of GABA-glycine was delivered during the presentation of a train of auditory clicks, an enhanced clickevoked response was apparent at the drug offset (Fig. 8B). Of 57 neurons that were inhibited by GABA-glycine pulses, 35 (61\%) exhibited an enhanced discharge rate at the termination of the drug delivery. Of 19 neurons to which either GABA or glycine was applied, 15 (79\%) exhibited an enhanced discharge rate. GABA-glycine application in the absence of excitatory synaptic drive did not produce an enhanced discharge rate.

\section{DISCUSSION}

Electrophysiological studies of sound localization mechanisms have considered the role of inhibition during relatively short stimuli (typically no more than $100 \mathrm{msec}$ ), usually consisting of steady-state level or phase differences between the two ears. Under these conditions the responses of many neurons in the central auditory pathway are tuned to ITD or ILD (Rose et al., 1966; Goldberg and Brown, 1969; Brugge et al., 1969; Boudreau and Tsuchitani, 1970; Brugge and Merzenich, 1973). The present study has revealed that dynamic ILD stimuli elicit responses that are not predicted on the basis of the response to static binaural stimuli. For many IC neurons, the response to a given ILD stimulus was enhanced if that stimulus was immediately preceded by a dynamic reduction in level at the ipsilateral inhibitory ear (i.e., a dynamic ILD). This was termed conditioned enhancement. When acoustic stimulation of the ipsilateral inhibitory ear was increased suddenly, many IC neurons displayed a smaller than expected response at the new binaural stimulus, termed conditioned suppression.

Similar responses have been reported previously for a variety of 


\section{Spontaneous Ipsi Modulation}
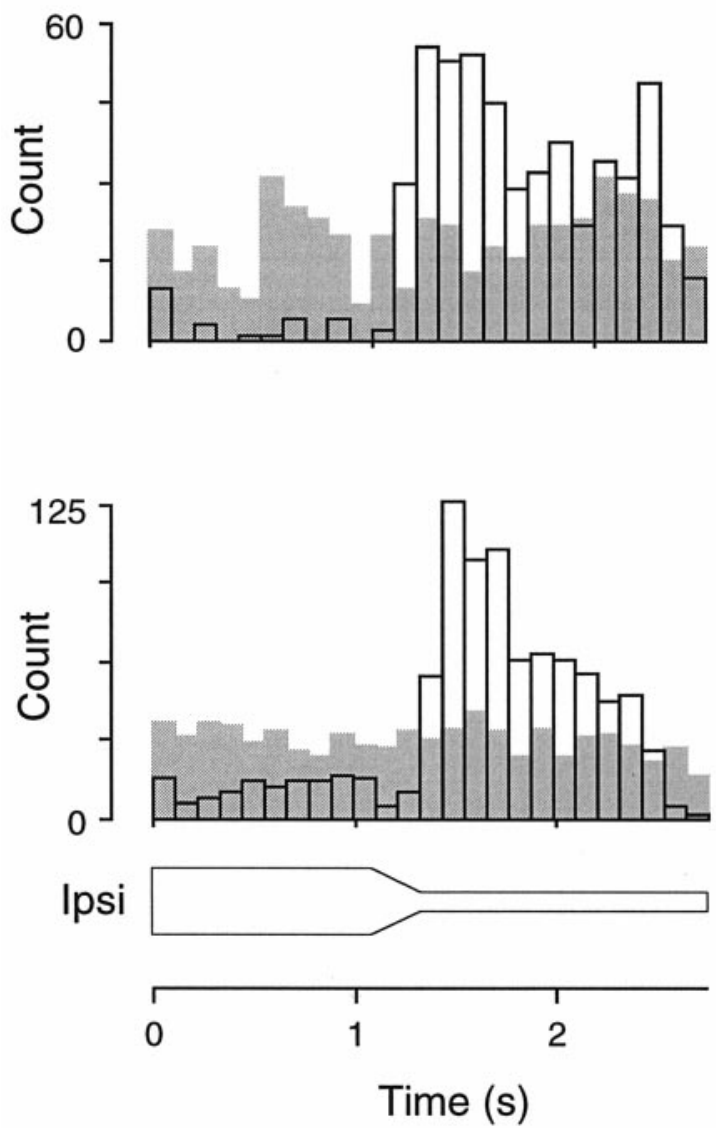

Figure 6. When IC neurons were firing spontaneously, it was possible to test the effect of ipsilateral stimulation alone. In both neurons shown, the spontaneous discharge level was enhanced dramatically by decreasing sound level at the ipsilateral ear.

virtual acoustic motion stimuli (Sovijärvi and Hyvärinen, 1974; Yin and Kuwada, 1983; Wagner and Takahashi, 1990; Spitzer and Semple, 1991, 1993; Ahissar et al., 1992; Stumpf et al., 1992; Takahashi and Keller, 1992; Toronchuk et al., 1992). In the barn owl, midbrain neurons respond selectively to the direction of virtual motion stimuli, although the absolute discharge rates seldom exceeded the response to static stimulation, perhaps because of the transient nature of the excitatory stimulus or the presence of inhibitory sidebands (Wagner and Takahashi, 1992). Neuronal responses to the unfavorable direction of motion did suppress discharge rates below the static response, similar to conditioned suppression in the gerbil IC. In the auditory cortex, moving stimuli can produce either enhanced or suppressed discharge rates in some neurons, compared with stimuli from a static sound source (Ahissar et al., 1992).

The present results suggest that the conditioning of responses by dynamic stimulation depends on several stimulus parameters, including the depth, rate, exposure period, and target level of the stimulus. When the stimulus parameters are optimized, the dynamically conditioned responses are often found to last for several seconds after the virtual motion component of the stimulus. Both the acoustic and pharmacological experiments suggest that an aftereffect of synaptic inhibition transiently enhances or sup- presses the gain of excitatory afferents in the IC. The following discussion considers the stimulus parameters necessary to observe these phenomena, possible underlying cellular mechanisms, and the possible relevance to processing of dynamic stimuli, such as acoustic motion.

\section{Acoustic parameters that condition neural responses}

Conditioned enhancement and suppression were observed under several stimulus conditions in this study. The first requirement was stability of the contralaterally evoked response over seconds of stimulation. Despite clear evidence of ILD sensitivity shaped by ipsilateral inhibition in response to $200 \mathrm{msec}$ binaural stimuli, several neurons failed to respond to dynamic stimuli. One prominent reason for this failure was a negligible response to constant monaural stimulation of the contralateral excitatory ear after $>1$ sec of stimulation. Therefore, it remains possible that these nonresponding neurons might have displayed sensitivity to virtual motion stimuli if the contralateral stimulus had been optimized. Although long-duration auditory stimuli are rarely used, a complete cessation of the sound-driven response is almost certainly a CNS mechanism. Recordings from single cat auditory nerve fibers during discontinuous tonal stimuli of $32 \mathrm{sec}$ duration demonstrate prominent adaptation but not cessation of activity (Javel, 1996). Habituation along the ipsilateral pathway may also preclude a response to dynamic stimulation, but examination of this possibility will require analysis of inhibitory synaptic currents (Covey et al., 1996). At this juncture, it might reasonably be asked whether the anesthetic schedule concealed or precipitated the conditioning influence of responses to virtual motion stimuli. Although full resolution must await replication in an unanesthetized preparation, conditioned enhancement and suppression were observed under three different anesthetic schedules in the present study, including a nonbarbiturate protocol (see Materials and Methods).

For neurons that fulfilled these minimum response criteria, it was found that the unexpectedly high or low discharge rates were limited to a narrow range of virtual motion stimuli. Figure 2 illustrates this point by comparing the response elicited by a particular binaural level combination under three conditions: immediately after (1) an increasing, (2) a decreasing, or (3) a constant (control) ipsilateral stimulus level. In all cases, the contralateral level remained constant throughout. If a neuronal response to these binaural level stimuli maintained a consistent relation to the ILD, with no particular dependence on the recent history of stimulation, these curves should be identical. To the extent that the initial steady-state binaural component and the virtual motion component affect subsequent responses, these three curves diverge. For $65 \%$ of EI neurons tested, decrease of ipsilateral level led to a larger than expected discharge rate in the succeeding $1 \mathrm{sec}$ interval. This effect typically was limited to a specific range of ipsilateral levels for a given contralateral level, usually those that fell between the middle and tail of the ILD function (Figs. 1, 2). An analogous finding has been made for low $\mathrm{CF}$ neurons in the inferior colliculus of gerbils and cats using a dynamic interaural phase delay stimulus (Semple and Spitzer, 1991, 1993). Modulation of interaural phase near the edge of static ITD functions was most effective at producing unexpectedly large or small responses. Presumably these sensitive portions of the ILD and ITD static tuning curves are associated with overlapping excitatory and inhibitory influences.

Variation of two stimulus parameters, the rate of ipsilateral modulation and the depth of ipsilateral modulation, was found to affect the amplitude of conditioned enhancement. In general, 


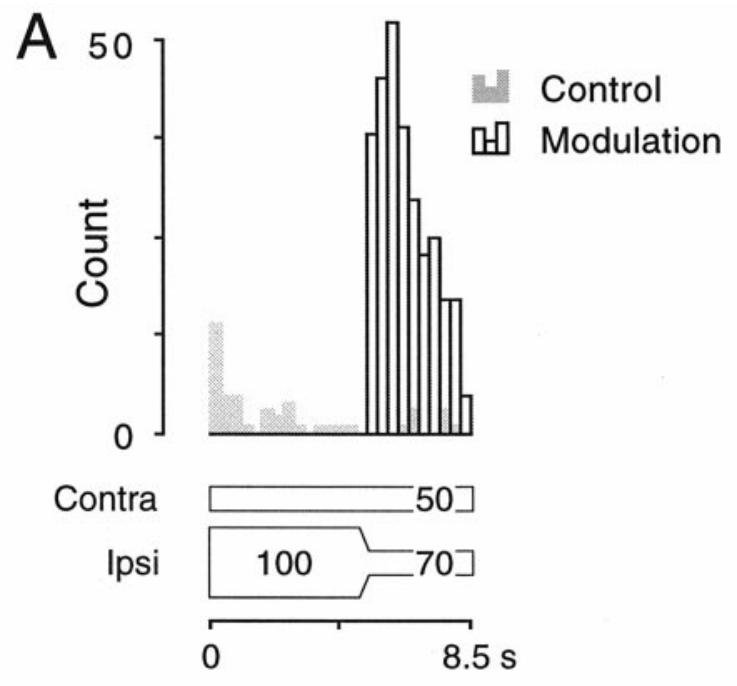

B
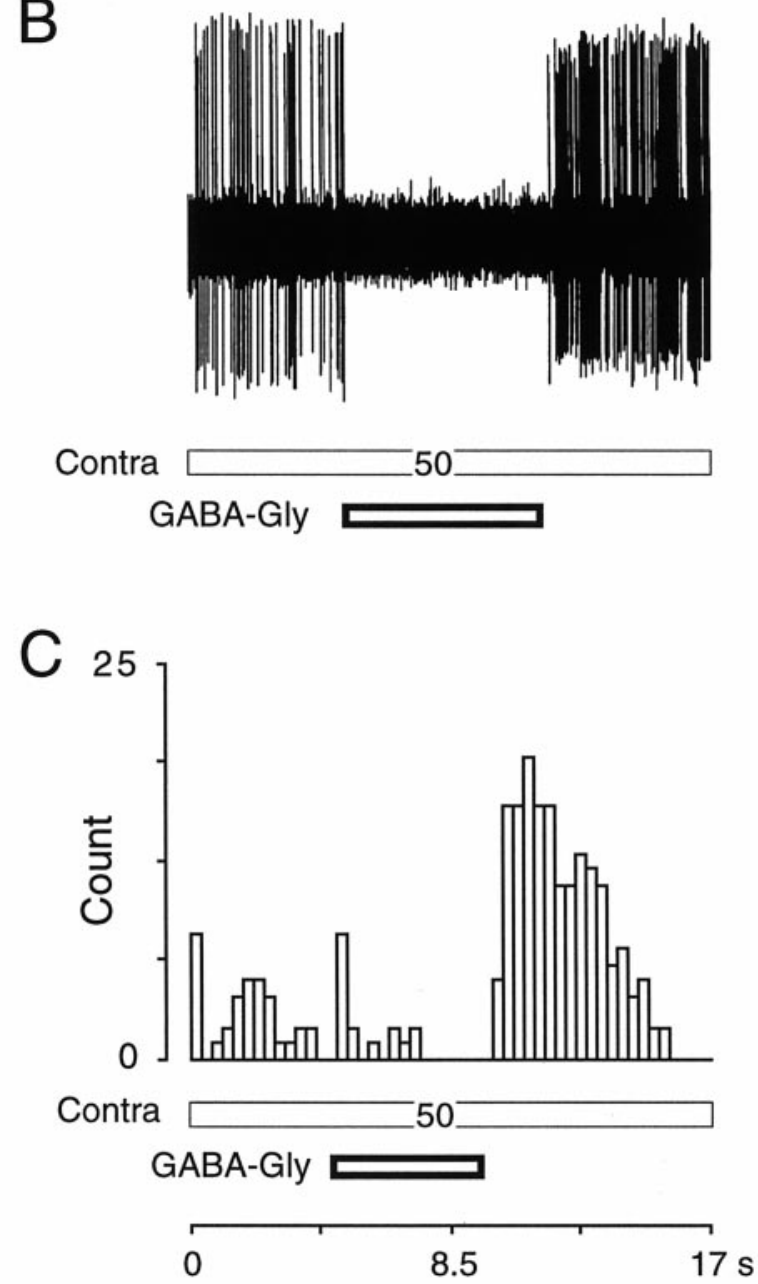

Figure 7. Enhanced discharge may be elicited by exposure to an indirect (acoustic) or direct (pharmacological) inhibitory conditioning stimulus. $A$, Compared with the control run (gray bars), in which a steady-state binaural stimulus was delivered, the neuron displayed an enhanced response after a virtual motion stimulus (open bars), $5 \mathrm{sec}$ after stimulus onset. (SPL values are shown on stimulus bars.) $B$, The same neuron was tested with a pulse of GABA-glycine from the recording pipette. The oscilloscope trace reveals that suppression of the contralaterally evoked response was complete during the drug delivery, and that the quality of modulation of ipsilateral level by $\pm 10-20 \mathrm{~dB}$ produced the largest changes in discharge rate, and the response seemed saturated by $30 \mathrm{~dB}$ (Fig. 4). This finding suggests that the magnitude of the dynamic conditioning effect was proportional to the magnitude of synaptic inhibition. Conditioned enhancement was also found to rely on modulation rate. Periods of very slow modulation lasting up to $6 \mathrm{sec}$ (e.g., depth of $-30 \mathrm{~dB}$ at $5 \mathrm{~dB} / \mathrm{sec}$ ) were sufficient to produce an enhanced discharge rate compared with control trials (Fig. 5). However, more rapid modulation generally elicited greater maximum discharge rates. The most effective modulation period was typically $\sim 0.5 \mathrm{sec}$ (e.g., depth of $-30 \mathrm{~dB}$ at $60 \mathrm{~dB} / \mathrm{sec}$ ).

\section{Possible cellular mechanisms}

In the central auditory system, the influence of inhibitory afferents in shaping the frequency, intensity, or temporal selectivity of a neuron has been well described (Greenwood and Maruyama, 1965; Rose et al., 1966; Fujita and Konishi, 1991). Three lines of evidence suggest that conditioned enhancement in the IC is at least partially attributable to a postsynaptic mechanism. First, contralaterally evoked synaptic activity is not required. Modulation of the ipsilateral pathway alone is sufficient to increase spontaneous discharge rates in some IC neurons (Fig. 6). Although this does not imply that the excitatory afferents are irrelevant to the magnitude of response, it does demonstrate the primacy of synaptic inhibition.

Second, the contralaterally evoked discharge rate can be enhanced without actually using the presynaptic inhibitory terminals. When GABA-glycine were administered from the recording electrode, the contralaterally evoked discharge rate was enhanced immediately after the brief $(0.5-10 \mathrm{sec})$ drug exposure (Figs. 7, 8). The effect of inhibitory transmitter was also observed for individual application of GABA or glycine, and $\sim 60 \%$ of neurons that were inhibited during drug delivery also exhibited enhanced discharge at the offset of delivery. This experiment demonstrates that the electrical properties of the IC neuron can be modified transiently by GABAergic or glycinergic inhibition.

Third, conditioned enhancement does not appear to result from inhibitory fatigue. If the inhibitory afferents became weaker with continued use, then one would predict that the discharge rate would increase gradually even during the more intense phase of inhibitory stimulation. The opposite was true (Fig. $1 C$ ); as ipsilateral sound level was continuously modulated, the suppression of spontaneous activity remained prominent during the interval when level was greatest (e.g., when ipsilateral level was $60 \mathrm{~dB}$ in Fig. $1 C$ and $30 \mathrm{~dB}$ in Fig. $1 E$ ). Therefore, we hypothesize that synaptic inhibition may induce a change in the postsynaptic neuron that is secondary to hyperpolarization. This hypothesis may be tested in future experiments with GABA-glycine receptor antagonists in vivo or with direct perturbation of membrane potential in the IC brain slice preparation (Lo et al., 1998).

The prolonged time course of conditioned enhancement (Fig. 3) distinguishes it from the well-described cellular property, anode break excitation, that constitutes a momentary decrease in sodium channel inactivation (Hodgkin and Huxley, 1952). One possible cellular mechanism in IC neurons could involve a membrane hyperpolarization of sufficient magnitude and duration to activate an inward current, resulting in long-lasting membrane

$\leftarrow$

the recording did not suffer during drug ejection. $C$, Several GABA-glycine trials accumulated by delivering the GABA-glycine at a discrete latency to the onset of the contralateral excitatory stimulus $(E=50 \mathrm{~dB}$ SPL $)$. The neuron displayed a dramatically enhanced response at the offset of the drug pulse, consistent with its acoustically evoked properties (unit 96B017.006). 


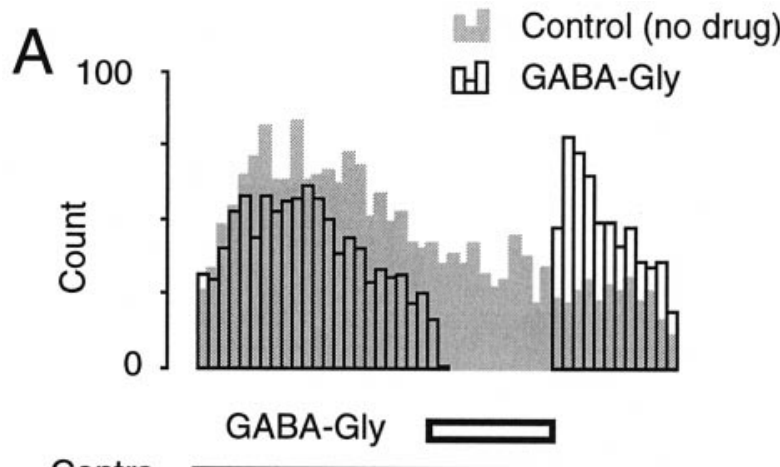

Contra
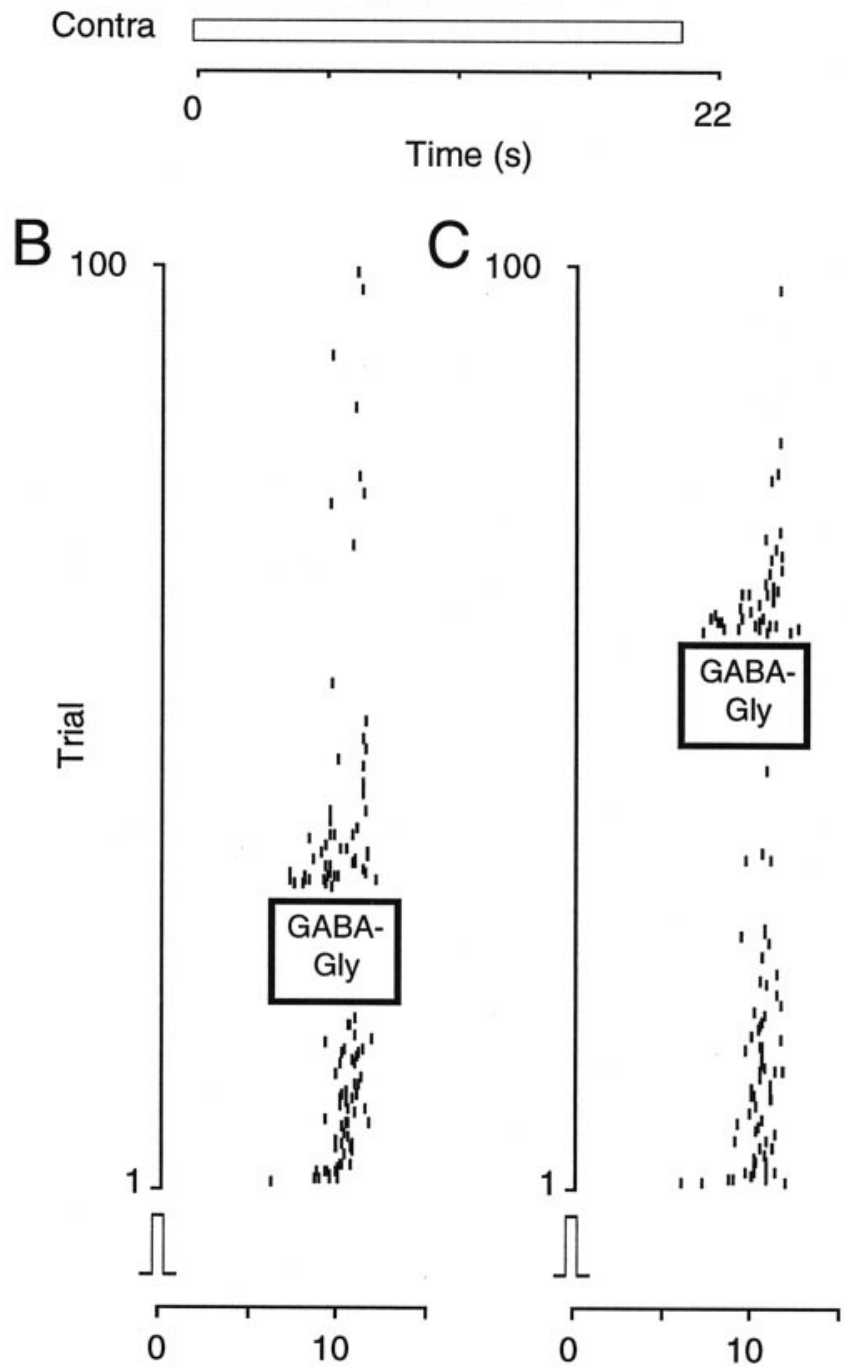

Time (ms)

Figure 8. Conditioned enhancement from pharmacological inhibition was present in a wide range of IC neurons. $A$, When stimulated with a contralateral tone $(60 \mathrm{~dB}$ SPL) for $20 \mathrm{sec}$, an IC neuron displayed a vigorous response (gray bars). When a GABA-glycine pulse was delivered after $10 \mathrm{sec}$, discharge was blocked transiently but returned to greater than control values at the pulse offset (open bars) (unit 96B028.003). B, When stimulated with contralateral clicks at $50 \mathrm{~Hz}$, a different neuron displayed a decreasing response, but when a $2 \mathrm{sec}$ pulse of GABA-glycine was delivered (left), the response reappeared at the pulse offset. $C$, The GABA-glycine pulse was delivered later in the trial during a second run (right) (unit 96B027.003). depolarization. Currents of this sort have been identified in cardiac muscle, photoreceptors, spinal ganglia, and sensorimotor cortex (Yanagihara and Irisawa, 1980; Mayer and Westbrook, 1983; Bader and Bertrand, 1984; Spain et al., 1987). It also remains possible that $\mathrm{GABA}_{\mathrm{A}}$ receptors mediate an unexpected synaptic response in the IC. For example, we found recently that muscimol produced a transient hyperpolarization, followed by a longer-lasting depolarization in IC neurons in vitro, and this was accompanied by a rise in intracellular free calcium (Lo et al., 1998). Finally, it is conceivable that the continuous (i.e., 1-2 sec) presence of inhibitory transmitter could desensitize the postsynaptic receptors or activate inhibitory autoreceptors, either of which might decrease net inhibitory strength. This mechanism could only explain our results if contralateral stimuli also recruited inhibitory transmission, down-regulation of which would produce an increase in contralaterally evoked discharge.

\section{Functional significance}

In this study we have applied a dynamic acoustic stimulus that is based on modulation of a binaural cue for sound localization, and consequently much of our discussion is phrased in terms of virtual acoustic motion. Nevertheless, it is important to consider the more general implication that time-varying ITD and ILD stimuli might be two instances of a general class of dynamic stimuli that have the potential to condition the responsiveness of central auditory neurons. In this context, the common characteristic may be the interplay between excitatory and inhibitory synaptic influences, including modifications of synaptic strength that may occur during the course of stimulation.

Although previous reports have not tested the delayed effects of inhibitory transmission on excitatory gain directly, there are many findings that might be explained by this mechanism. For example, several groups have demonstrated that discharge rate is enhanced by time-varying acoustic signals, possibly because of modulation of inhibitory synaptic drive (Spitzer and Semple, 1991, 1993; Ahissar et al., 1992; Takahashi and Keller, 1992). For example, the discharge rates of primate auditory cortex neurons were most enhanced by moving stimuli that entered the contralateral field, presumably leaving a region of inhibition (see Ahissar et al., 1992, their Fig. 3). In the auditory cortex, selective recruitment of inhibitory afferents within a delimited frequency band has also been shown to produce conditioned enhancement (Phillips and Hall, 1992).

Conditioned enhancement may also help explain computational properties in other regions that have previously been ascribed to sensory adaptation. For example, the response-contrast curves of visual cortical neurons shift laterally along the contrast axis after preexposure at a specific contrast level, thus providing a mechanism for gain control (Ohzawa et al., 1985). At the synaptic level, conditioned enhancement may provide a mechanism for adjusting the gain of excitatory afferents, particularly during the course of prolonged acoustic stimuli.

\section{REFERENCES}

Adams JC, Mugnaini E (1984) Dorsal nucleus of the lateral lemniscus: a nucleus of GABAergic projection neurons. Brain Res Bull 13:585-590.

Ahissar M, Ahissar E, Bergman H, Vaadia E (1992) Encoding of soundsource location and movement: activity of single neurons and interactions between adjacent neurons in the monkey auditory cortex. J Neurophysiol 67:203-215.

Bader CR, Bertrand D (1984) Effect of changes in intra- and extracellular sodium on the inward (anomalous) rectification in salamander photoreceptors. J Physiol (Lond) 347:611-631.

Boudreau JC, Tsuchitani C (1970) Cat superior olive s-segment cell 
discharge to tonal stimulation. In: Contributions to sensory physiology, Vol 4 (Neff WD, ed), pp 143-213. New York: Academic.

Brugge JF, Dubrovsky NA, Aitkin LM, Anderson DJ (1969) Sensitivity of single neurons in auditory cortex of cat to binaural tonal stimulation: effects of varying interaural time and intensity. $\mathrm{J}$ Neurophysiol 32:1005-1024.

Brugge JF, Anderson DJ, Aitkin LM (1970) Responses of neurons in the dorsal nucleus of the lateral lemniscus of cat to binaural stimulation. J Neurophysiol 33:441-458.

Brugge JF, Merzenich MM (1973) Responses of neurons in auditory cortex of the macaque monkey to monaural and binaural stimulation. J Neurophysiol 36:1138-1158.

Cai H, Carney LH, Colburn HS (1997) A model for binaural response properties of inferior colliculus neurons: II. A model with ITDsensitive excitatory and inhibitory inputs and an adaptation mechanism. $\mathrm{J}$ Acoust Soc Am, in press.

Casseday JH, Ehrlich D, Covey E (1994) Neural tuning for sound duration: role of inhibitory mechanisms in the inferior colliculus. Science 264:847-850.

Covey E, Kauer JA, Casseday JH (1996) Whole-cell patch-clamp recording reveals subthreshold sound-evoked postsynaptic currents in the inferior colliculus of awake bats. J Neurosci 16:3009-3018.

Faingold CL, Anderson CA, Randall ME (1993) Stimulation or blockade of the dorsal nucleus of the lateral lemniscus alters binaural and tonic inhibition in contralateral inferior colliculus neurons. Hearing Res 69:98-106.

Fujita I, Konishi M (1991) The role of GABAergic inhibition in processing of interaural time difference in the owl's auditory system. J Neurosci 11:722-739.

Goldberg JM, Brown PB (1969) Responses of binaural neurons of dog superior olivary complex to dichotic tonal stimuli: some physiological mechanisms of sound localization. J Neurophysiol 32:613-636.

Greenwood DD, Maruyama N (1965) Excitatory and inhibitory response areas of auditory neurons in the cochlear nucleus. J Neurophysiol 28:863-892.

Harnischfeger G, Neuweiler G, Schlegel P (1985) Interaural time and intensity coding in superior olivary complex and inferior colliculus of the echo locating bat Molossus ater. J Neurophysiol 53:89-109.

Hodgkin AL, Huxley AF (1952) The dual effect of membrane potential on sodium conductance in the giant axon of Loligo. J Physiol 116:497-506.

Javel E (1996) Long-term adaptation in cat auditory nerve fiber responses. J Acoust Soc Am 99:1040-1052.

Le Beau FEN, Rees A, Malmierca MS (1996) Contribution of GABAand glycine-mediated inhibition to the monaural temporal response properties of neurons in the inferior colliculus. $J$ Neurophysiol 75:902-919.

Lo Y-J, Rao SC, Sanes DH (1998)Modulation of calcium by inhibitory systems in the developing auditory system. Neuroscience, in press.

Mayer ML, Westbrook GL (1983) A voltage-clamp analysis of inward (anomalous) rectification in mouse spinal sensory ganglion neurones. J Physiol (Lond) 340:19-45.

Merchan MA, Saldana E, Plaza I (1994) Dorsal nucleus of the lateral lemniscus in the rat: concentric organization and tonotopic projection to the inferior colliculus. J Comp Neurol 342:259-278.

Mogdans J, Knudsen EI (1994) Representation of interaural level difference in the VLVp, the first site of binaural comparison in the barn owl's auditory system. Hearing Res 74:148-164.

Moore MJ, Caspary DM (1983) Strychnine blocks binaural inhibition in lateral superior olivary neurons. J Neurosci 3:237-247.

Nelson PG, Erulkar SD (1963) Synaptic mechanisms of excitation and inhibition in the central auditory pathway. J Neurophysiol 26:908-923.

Ohzawa I, Sclar G, Freeman RD (1985) Contrast gain control in the cat's visual system. J Neurophysiol 54:651-667.

Park TJ, Pollak GD (1993a) GABA shapes sensitivity to interaural intensity disparities in the mustache bat's inferior colliculus: implications for encoding sound location. J Neurosci 13:2050-2067.

Park TJ, Pollak GD (1993b) GABA shapes a topographic organization of response latency in the mustache bat's inferior colliculus. J Neurosci 13:5172-5187.

Park TJ, Pollak GD (1994) Azimuthal receptive fields are shaped by GABAergic inhibition in the inferior colliculus of the mustache bat. J Neurophysiol 72:1080-1102.

Phillips DP, Hall SE (1992) Multiplicity of inputs in the afferent path to cat auditory cortex neurons revealed by tone-on-tone masking. Cereb Cortex 2:425-433.

Reale RA, Brugge JF (1990) Auditory cortical neurons are sensitive to static and continuously changing interaural phase cues. J Neurophysiol 64:1247-1260.

Rose JE, Gross NB, Geisler CD, Hind JE (1966) Some neural mechanisms on the inferior colliculus of the cat which may be relevant to localization of a sound source. J Neurophysiol 29:288-314.

Saint Marie RL, Baker RA (1990) Neurotransmitter-specific uptake and retrograde transport of $\left[{ }^{3} \mathrm{H}\right]$ glycine from the inferior colliculus by ipsilateral projections of the superior olivary complex and nuclei of the lateral lemniscus. Brain Res 524:244-253.

Sanes DH (1990) An in vitro analysis of sound localization mechanisms in the gerbil lateral superior olive. J Neurosci 10:3494-3506.

Schwartz IR (1992) The superior olivary complex and lateral lemniscal nuclei. In: The mammalian auditory pathway: neuroanatomy (Webster DB, Popper AN, Fay RR, eds), pp 117-167. New York: Springer.

Shneiderman A, Chase MB, Rockwood JM, Benson CG, Potashner SJ (1993) Evidence for a GABAergic projection from the dorsal nucleus of the lateral lemniscus to the inferior colliculus. J Neurochem 60:72-82.

Sovijärvi ARA, Hyvärinen J (1974) Auditory cortical neurons in the cat sensitive to the direction of sound source movement. Brain Res 73:455-471.

Spain WJ, Schwindt PC, Crill WE (1987) Anomalous rectification in neurons from cat sensorimotor cortex in vitro. J Neurophysiol 57:1555-1576.

Spitzer MW, Semple MN (1991) Interaural phase coding in auditory midbrain: influence of dynamic stimulus features. Science 254:721-724.

Spitzer MW, Semple MN (1993) Responses of inferior colliculus neurons to time-varying interaural phase disparity: effects of shifting the locus of virtual motion. J Neurophysiol 69:1245-1263.

Stumpf E, Toronchuk JM, Cynader MS (1992) Neurons in cat primary auditory cortex sensitive to correlates of auditory motion in threedimensional space. Exp Brain Res 88:158-168.

Suga N (1988) Auditory neuroethology and speech processing: complexsound processing by combination-sensitive neurons. In: Auditory function: neurobiological basis of hearing (Edelman GM, Gall WE, Cowan WM, eds), pp 679-720. New York: Wiley.

Takahashi TT, Keller CH (1992) Simulated motion enhances neuronal selectivity for a sound localization cue in background noise. J Neurosci 12:4381-4390.

Toronchuk JM, Stumpf E, Cynader MS (1992) Auditory cortex neurons sensitive to correlates of auditory motion: underlying mechanisms. Exp Brain Res 88:169-180.

Wagner H, Takahashi TT (1990) Neurons in the midbrain of the barn owl are sensitive to the direction of apparent acoustic motion. Naturwissenschaften 77:439-442.

Wagner H, Takahashi T (1992) Influence of temporal cues on acoustic motion-detection sensitivity of auditory neurons in the owl. J Neurophysiol 68:2063-2076.

Wu SH, Kelly JB (1996) In vitro brain slice studies of the rat's dorsal nucleus of the lateral lemniscus. III. synaptic pharmacology. J Neurophysiol 75:1271-1282.

Yanagihara K, Irisawa H (1980) Inward current activated during hyperpolarization in the rabbit sinoatrial node cell. Pflügers Arch 385:11-19.

Yin TCT, Kuwada S (1983) Binaural interactions in low-frequency neurons in inferior colliculus of the cat. II. effects of changing rate and direction of interaural phase. J Neurophysiol 50:1000-1019.

Zook JM, Casseday JH (1987) Convergence of ascending pathways at the inferior colliculus of the mustache bat, Pteronotus parnellii. J Comp Neurol 261:347-361. 